\title{
LOS GRUPOS DE DISCUSIÓN, UNA ESTRATEGIA DISCURSIVA PARA EL ARTE
}

Un día venía un ciempiés caminando sin pensar en nada. Por el mismo camino, en sentido contrario, venía una araña. La araña al ver al ciempiés se hizo a un lado y le preguntó: ¿Cómo haces para caminar con tantas patas? ¿Cómo coordinas?

¿Mueves primero los pies de la izquierda y después los de la derecha? ¿O mueves un pie derecho después uno izquierdo? ¿O levantas el cuerpo para mover un par de tus pies? El ciempiés entonces se puso a pensar para responderle a la araña y ya no se movió nunca más.

Juan Gelman

Este artículo re-crea la manera como fueron configurados tres grupos de discusión que conversaron en torno a las prácticas artísticas contemporáneas, en el marco del proyecto de investigación Asomacandela, llevado a cabo por profesores integrantes del Grupo De Humanitate adscrito a la Facultad de Humanidades y Ciencias Sociales de la Universidad Javeriana Cali. La configuración de escenarios posibles al interior de entramados diversos, provocaría la múltiple mirada al asunto para que las nuevas prácticas no se asuman simplemente como objeto de conocimiento, sino como espacios para el pensamiento. Atendiendo la metodología propuesta por Jesús Ibáñez denominada Grupos de Discusion ${ }^{2}$ se produjeron tres situaciones de discurso -durante el transcurso de la investigación-, entendidas como el conjunto de circunstancias en medio de las cuales se desarrolla un acto de enunciación oral ${ }^{3}$ por parte de una

Florencia Mora y Victor Martínez Profesores del Departamento de Humanidades Universidad Javeriana Cali

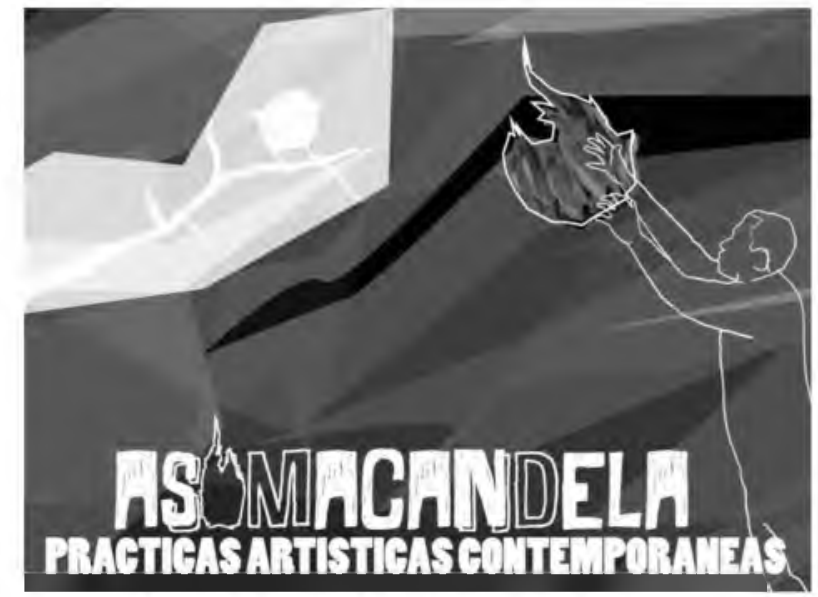

selección de actuantes, incluyendo a los investigadores. Durante tres sesiones, artistas y colectivos de artistas provenientes de diferentes espacios (gestión, creación, crítica) interactuaron a partir de preguntas generadoras respecto a la tensión política, epistémica, estética, académica, económica que desafían las llamadas prácticas artísticas contemporáneas.

Es un desafío para los artistas encontrar metodologías investigativas congruentes con sus prácticas que les ayude a avanzar y no les haga quedar inmóviles en la mitad del camino. Las distintas técnicas de investigación presentan límites enmarcados en epistemologías determinadas y, a la vez que permiten develar ciertos aspectos de la realidad, ocultan otros. Así pues, no es simple la aproximación a este campo de conocimiento ya que la naturaleza de las prácticas artísticas exige imaginar y construir caminos de indagación pertinentes para

1- Juan Gelman, poeta contemporáneo, ganador de varios de los galardones literarios de mayor prestigio en el mundo como el Premio Cervantes 2007 y el Reina Sofía de Poesía Iberoamericana.

2 - Ibáñez, Jesús. "Más allá de la sociología. El Guipo de discusión: Técnica y Crítica. Siglo Veintiuno de España Editores. Madrid 2003.

3 - Ibíd. Pág.262. Este acto de carácter oral, puede además expresarse en forma escrita. 
avanzar en la producción y creación de nuevas experiencias estéticas sin descuidar el rigor y la flexibilidad, al mismo tiempo. ¿Cómo investigar los posibles sentidos que implican las prácticas artísticas contemporáneas desde las dinámicas políticas, contextuales, socioculturales y pedagógicas a partir de las cuales éstas toman forma? "Asomacandela, prácticas artísticas contemporáneas" asumió este desafío desde los Grupos de Discusión o la puesta en escena de contextos existenciales de discurso.

En "Asomacandela", propuesta de investigación del grupo De Humanitate, la pregunta no radicó en la descripción de las prácticas o en la búsqueda de su reconocimiento mediante la sustentación de un discurso. Más bien se quiso desde la experiencia, asumir la investigación como un proceso de presencia en el que los actuantes encontraran aquello distinto a lo que proyectaban, para que los invadiera su experiencia y les hiciera decir alguna cosa (Larrosa), porque conocer no es capturar, aprehender 0 explicar, sino experimentar a partir de la (sobre) vivencia. Por ello, se propuso la organización de grupos de discusión que interactuaran conformando escenarios discursivos; más allá de generar una expresión artística o un devenir de sucesos que decantaran en experiencia estética, los grupos de discusión propuestos pretendieron serpor sí mismos - una propuesta estética.

Más allá de un simple documento escrito o un audiovisual que recogiera las actuaciones del discurso, concebidos como producto del proceso, el producto del proyecto consistía en crear una práctica artística que interrogara los modos como se investiga, que transgrediera cánones establecidos, integrando a la investigación los saberes espontáneos, las actitudes, los gestos, los textos y los objetos, elementos todos que conformaban la situación de discurso.

Ahora bien, antes de describir los procedimientos de la metodología, es importante señalar, que el grupo de discusión, más que una técnica, debe ser considerada una práctica investigativa (Callejo, 2001) óptima para recoger las experiencias y discursos de los sujetos y sus relaciones, entre sí, con el contexto y con los objetos donde acontece la producción artística. Ello supone diseñar y realizar una experiencia para comprender otras experiencias que en su densidad trascienden la aplicación o utilización instrumental de una técnica. En tal sentido, la multiplicidad de discursos que produce el colectivo va más allá de lo fenomenal y se ofrece en nuevas formas al entendimiento del investigador.

Los grupos de discusión son propios de las investigaciones cualitativas ya que permiten revelar significados producidos en el devenir del discurso grupal, sus silencios, sus entonaciones, sus percepciones, sus adhesiones, sus reiteraciones 0 sus disensos, los cuales difícilmente podrían ser incluidos de otra forma: "perspectiva de actores" (Callejo, 2001). "Frente a los presupuestos teóricos que fundamentan el método cuantitativo, el cualitativo parte del supuesto básico de que el mundo social está construido con significados y símbolos". (Llopis G, 2004, pág. 23). De tal forma, esta práctica investigativa ayuda a visibilizar 10 particular, lo cotidiano, dimensionar lo espontáneo, identificar la estructura de los procesos y los sentidos más recurrentes en tiempos y espacios específicos, características presentes en una práctica artística viva.

En términos descriptivos, "Javier Callejo (2001: 6576) ha realizado una interesante caracterización situacional del grupo de discusión... es una situación pública de interacción face-to-face que supone un encuentro entre personas que no se conocen y que van a verse envueltas en un proceso de reagrupación y lucha de intereses e identidades, coordinados por un moderador, en un tiempo y un espacio y a partir de una demanda de información..." (Llopis G, 2004, pág. 28). Los participantes acceden libremente a conversar en un pequeño grupo sobre una determinada experiencia de su interés, se disponen a encontrarse con sus saberes y conocimientos en una lógica permeable, flexible, y con el ánimo de aportar lo propio.

Aunque la grupalidad es una característica clave en esta práctica, su sentido colectivo no es lo que garantiza la calidad de la información, del proceso, ni de los resultados. Actualmente, los grupos de discusión toman distancia de los grupos focales, las entrevistas grupales, terapias grupales y otras actividades colectivas que hacia mediados del siglo anterior tuvieron auge. Con los grupos de discusión 
se habla de un cierto eclecticismo necesario "ya que se desarrolla en un proceso de incesante adaptación a las exigencias de las situaciones de estudio" (Llopis G, 2004, pág. 26) y a su manera han ido perfilando una manera propia de producir y analizar información.

Algunos suelen confundir los grupos de discusión con los grupos focales, quizá porque con Merton, Fiske y Kendall en The Focused Interview (1956) se señalaron algunos antecedentes comunes que con el tiempo fueron diferenciándose más claramente; por ello vale la pena puntualizar que mientras los grupos focales son positivistas, sirven al control social, son experimentales, evalúan productos comunicativos, conciben las palabras por su significado, comprenden el lenguaje como un mecanismo de estímulorespuesta, tienen un enfoque cerrado sobre el problema, parten del individuo, generan una información específica y detallada, y suponen una moderación directiva, los grupos de discusión se mueven en un paradigma dialéctico-estructural, están al servicio de la transformación-liberación social, se fundan en la reflexión, pretenden comprender procesos sociales, conciben los significados de las palabras en contextos de sentido, comprenden el lenguaje como un juego de conversación, tienen un enfoque interdisciplinar y abierto sobre el problema, parten de la producción grupal, generan una información más estructural y comprensiva, e implican una moderación no-directiva (David.B, 2007).

Ibáñez (1979) el principal investigador que ha fundamentado esta práctica, critica algunas técnicas grupalistas aparentemente liberadoras (del cuerpo, de la palabra, etc.) por su carácter colectivo, que sin embargo, se concentran en recolectar datos sin atreverse a cuestionar el origen enfermo del capitalismo y sus efectos sobre los individuos, las sociedades y los discursos, convirtiéndolas en meros instrumentos de legitimación del consumo. "El discurso del grupo es el producto de una producción, no de una recolección: en el discurso queda memoria de las huellas de ese proceso. $Y$ no hay modo de evitarlo: no hay técnica inocente..." (pág. 261). Los grupos de discusión emergen entonces como un desafío por superar las dicotomías sociales, económicas, políticas y culturales, y promover una esperanza de vida mejor a través de la palabra y los grupos.

Los grupos de discusión suponen la producción de situaciones de discurso entendidas como un conjunto de circunstancias en medio de las cuales se desarrolla un acto de enunciación oral por parte de una selección de actuantes, incluyendo a los investigadores, como lo señala Ducrot (1972) citado por Ibáñez. Las circunstancias que conforman un grupo de discusión comprenden el entorno físico y social en el que se realiza este acto discursivo, y en el que intervienen activamente la imagen que tienen de él los interlocutores, la identidad de estos últimos, la idea que cada uno posee sobre el otro, incluyendo la representación que cada uno tiene sobre el pensamiento del otro. En esta especie de escenografías físicas y discursivas, se incluyen además los acontecimientos que han precedido al acto de enunciación, considerando especialmente las relaciones que han tenido hasta entonces los interlocutores y los intercambios de discursos donde ocurre la enunciación.

Habiendo reconocido el carácter ecléctico de esta práctica investigativa, Callejo (2001) señala como cada investigador se ha apoyado en distintas fuentes teóricas para avalar la rigurosidad de la misma. Así, Ibañez toma referencia principalmente del estructuralismo y el psicoanálisis; Muchelli, de la psicología humanista, Morgan del empirismo, mientras que otros encuentran referentes en la fenomenología, el análisis del discurso, la sociolingüística, y en modelos dialógicos, entre otros.

"Asomacandela", en particular, se caracterizó por el deseo de extender esta metodología hacia lugares diversos, interpelando estamentos, reformulando, insistiendo y provocando discursos renovados y directos. En suma, se trató de considerar a las artes como expresión viva, en todo su despliegue, asumiendo que estas no representaban un valor absoluto e inamovible. El grupo de discusión opera en un momento preciso; actúa para reconocerse y quebrantar los esquemas, sacude y agita con el fin de que la acción genere otras de la misma naturaleza en una cadena continua de interpretaciones y discursos; el grupo de discusión es visiblemente una confesión colectiva. 
La realización de los grupos de discusión exige un cuidadoso diseño (UCM, 2010): la planeación del conjunto de la investigación, la selección de los participantes, la producción de situaciones de discurso, entre otros, no son actos ingenuos sino una fina filigrana capaz de engendrar un potente discurso. Así mismo, se recomienda realizar más de un grupo de discusión con el propósito de contar con mayor información, elementos de análisis e interpretación.

Ibáñez señala que el grupo de discusión exige un diseño abierto, que sus participantes son seres concretos, son sujetos en proceso, que digieren y se transforman continuamente. Añade que el contexto existencial del discurso (es decir, la situación de discurso) es un proceso de producción, y el discurso de todo el grupo, es el producto de la investigación. Al respecto, sabemos que el diseño del grupo de discusión, comporta los siguientes aspectos: Una selección de actuantes (participantes), un esquema de actuación (un repertorio de cuestiones o preguntas), y una interpretación (del contenido captado inmediatamente). Así, explica Ibáñez, "el diseño se abre al azar al menos por tres puertas: en la selección de los actuantes, en el esquema de actuación y en la interpretación que hace el investigador" (Ibáñez, 1979, pág. 264.).

En la selección de actuantes, el investigador tiene en cuenta los criterios del muestreo estadístico (heterogeneidad y representatividad): el número de participantes, las relaciones que se van a producir en el grupo, la pertinencia del grupo y de cada participante (incluye en el grupo a quienes reproduzcan mediante su discurso relaciones relevantes y la competencia lingüística de los actuantes (que garantiza discursos diferentes). En la selección de los actuantes es preferible observar que cuanto más enfocada sea ésta, más definida será la información que emerja; quizás sí convenga enfocar la selección aun teniendo en cuenta que una selección desenfocada también informaría. Ibáñez advierte que el proceso de selección deja muchas puertas abiertas y que por eso, nunca se sabe quién llega y siempre puede venir alguien que sorprenda.

El esquema de actuación parte de la provocación del discurso. Hay una pro-vocación (dice Ibáñez) que se hace explícita de parte del preceptor (que es quien pro-pone el tema), considerando también que todos los elementos de la situación tienden a provocar los discursos (espacio/tiempo de reunión, composición del grupo).

Una vez el preceptor o moderador presente el tema, éste es abordado por el grupo. El preceptor (quien orienta y anima al grupo) no construye el espacio de las respuestas y tampoco el orden de la discusión: el grupo mismo determina qué se va a hablar y se da el orden; aunque se reconoce de antemano que el preceptor elabora el cuestionario o las preguntas provocadoras y por ello, su deseo se pone en discurso, lo que prevalece es el deseo de quienes contestan. De allí que Ibáñez diga que el grupo de discusión también resulta idóneo como fase previa en la elaboración de las preguntas de investigación para que en su redacción quede plasmado el deseo de quienes van a construir las respuestas (Ibáñez, 1979).

En la interpretación del contexto situacional y el contexto lingüístico, no hay ningún plan previo; a lo largo de la discusión hay operaciones de interpretación no solo por parte del preceptor sino a por parte de los participantes (actuantes). $Y$ todas estas interpretaciones se van diseminando gradualmente durante la producción del discurso, provocando que en cada momento interpretativo se instaure una relación abierta, un proceso que se ubica entre la empírea y la teoría. El investigador - es decir, quien orienta el grupo - es también un sujeto en proceso que experimenta modificaciones en la discusión y en la recopilación del proyecto. No es extraño pues, que el proceso de investigación así propuesto, capte los deseos, los intereses, las pulsiones, los gestos, durante el espacio/tiempo del grupo, mientras la discusión se mantenga.

Las fronteras de un grupo de discusión se determinan por su tamaño, su duración, la composición y las relaciones entre el preceptor (moderador) y el grupo. Conviene que los actuantes puedan hablar unos con otros y ello se garantiza en la adecuada escogencia del espacio; los actuantes no deben quedar ni muy juntos ni muy lejos, ni ser muchos o ser pocos. En su texto sobre esta metodología, Ibáñez pone de manifiesto a Bion cuando dice - respecto de los grupos terapéuticos - que "sólo si los individuos se acercan suficientemente unos a otros es posible dar 
una interpretación sin necesidad de gritar; de la misma manera es necesario que todos los miembros del grupo puedan comprobar elementos en los que se fundamentan las interpretaciones" (Ibáñez, 1979, pág. 267).

El grupo minimo, estaria conformado por cuatro participantes pero lo recomendable es que el grupo no tenga más de doce actuantes o diez. Una vez seleccionados y reunidos los actuantes, el grupo comienza a discutir desde el momento en que el preceptor lo señale, es el preceptor quien empieza y es el quien termina. Para lbáñez que el grupo de discusión "se empotra en la realidad como un paréntesis - puesta entre paréntesis de la realidad - : las urgencias reales exigen a los actuantes el conocimiento de la extensión de ese paréntesis (obligaciones laborales, familiares, etc.), y como en los cuentos medievales, es preciso que el deseo sepa el plazo que se le asigna para su realización" (Ibáñez, 1979, pág. 274). El grupo de discusión puede reunirse durante dos o tres horas aunque también la reunión puede durar dos o tres dias. En general, la duración de la discusión del grupo depende de las condiciones específicas del proyecto.

En el caso de "Ásomacandela", se vivenciaron tres grupos de discusión así: uno en la Habana (Cuba) en el marco de la Oncena Bienal de La Habana, el segundo en la vereda Guachinte (Valle del Cauca) y el tercero en el seno de la Universidad Javeriana de Cali; se contó con grupos pequeños como se ha recomendado "Los grupos de discusión deben ser lo suficientemente pequeños para permitir la oportunidad a cada participante de compartir su discernimiento de las cosas y a la vez lo suficientemente grande como para proveer diversidad de percepciones" (Bisquerra, 2009, pág. 344), y las sesiones duraron entre tres y cuatro horas. La selección de participantes tuvo en cuenta la heterogeneidad del grupo considerando la predeterminación de las clases de actuantes, su contexto de actuación y los posibles canales de relación topológica (Ibañez, 1979).

Algunos de ellos fueron contactados por medio de correo electrónico, otros por via telefónica y otros personalmente. Los grupos de discusión y sus participantes se relacionan en este texto; todos ellos fueron conscientes del objeto de estudio de esta investigación y expresaron su consentimiento para los registros respectivos. El diseño de las circunstancias 0 situaciones de discurso fue realizado cuidadosamente como se puede constatar en los registros fotográficos: los espacios, los dispositivos (mesas, manteles, sillas, tableros, marcadores, libretas, lapiceros, etc.), los alimentos y bebidas, y demás detalles que facilitaron la producción discursiva del colectivo. AsI mismo se contó con un guión de preguntas generadoras que pudieran ayudar a emerger lo que pensaban los participantes respecto a la tensión política, epistémica, estética, académica, económica de las prácticas artísticas. En tal sentido, la moderación fue un elemento clave para el desarrollo de los grupos de discusión puesto que la moderadora o preceptora ejerció un control suave sobre el grupo, estuvo en permanente vigilancia del transcurso de las intervenciones, de manera que sutilmente devolvía la discusión al objetivo original cuando este se desviaba por información irrelevante y de esa manera asegurar que el discurso permaneciera encarrilado (Llopis G, 2004).

Luego de la discusión, la preceptora, investigadora principal, abordó la fase de la interpretación; recogió la información, la descodificó e interpretó los discursos. En esta fase, el preceptor se enfrenta a la gran cantidad de información emanada de los discursos y ejerce su capacidad de interpretación que para Ibañiez tiene mucho que ver con la intuición. Al respecto el autor señala: "para poner orden en la masa de datos (seleccionar los pertinentes e integrarlos en un espacio teórico unitario debe ponerse en marcha su intuición (la del preceptor). La captación intuitiva (es decir, uno intuito de la unidad inmediatamente perceptible de una situación,) de un estilo de vida o de una manera de ser, conduce a indagar, en sus relaciones significantes, propiedades y relaciones que no se presentan

sino sucesivamente en el trabajo

analítico; este ejercicio de

captación actúa como escudo

protector frente al riesgo de

atomización del objeto que

resulta, por ejemplo, recurrir a indicadores impotentes, objetivar las manifestaciones de una actitud 0 de un ethos, fragmentándolas.

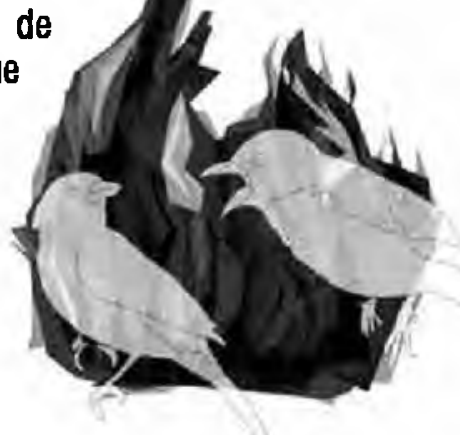


Esta capacidad de intuición es un precipitado de toda la experiencia y de todo el saber del investigador".

Agrega Ibáñez que la intuición, además de ser un proceso intelectual, es una actitud que implica las pulsiones del investigador, sus fantasmas y la situación que engloba la discusión. Por ello, en el proceso de interpretación se enfrentan las emociones, la incidencia del grupo de discusión en el preceptor y viceversa, el espacio empírico, los discursos y otras manifestaciones prosódicas, kinésicas, proxémicas, etc., el espacio teórico y la aplicabilidad de los datos a la teoría.

En síntesis, señala ibáñez que el grupo de discusión es una fábrica de discursos, en la que el preceptor tiene los hilos y dirige el proceso de producción del discurso. El preceptor no puede manifestar su autoridad en la producción de los discursos a no ser que se trate de una provocación que pretenda incentivar la palabra, dinamizando el proceso; los actuantes adoptan dos posturas: una postura crítica (son conscientes de la discusión) y poética (se saben y se reconocen como sujetos en proceso).

Esta experiencia de provocar situaciones de discurso en torno a las prácticas artísticas, busca ante todo el encuentro dinámico y afectivo, en la pretensión de "hacer grupo para no hacerlo", valerse de su estructura, deshaciéndola, reconociendo las experiencias previas que nos han traído a hablar de esto, de lo que decimos que hacemos, de lo que hacemos y lo que pensamos. Los grupos de discusión así propuestos, buscan las grietas de esas experiencias reinterpretando el decir de quienes estipulan y especulan lo que debemos ser como individuos y como grupo.

La palabra "grupo" proviene de la época del renacimiento y designa un concepto, que nada tiene que ver con el control social; la palabra italiana "groppo" se refiere a un conjunto de individuos que componen un tema plástico, y para la cultura germano occidental, "kruppa" significa redondo, nudo. En su texto, Ibañez plantea que quizás etimológicamente y siguiendo a Anzieu, la palabra grupo tenga que ver con lazo (nudo) por derivación, y con plenitud rebosante (redondo) cuyo paradigma es el pecho. En todo caso, el grupo formado en estos encuentros de Asomacandela se relaciona más con el convite, con la invitación, es decir, con un colectivo de individuos que componen una fratría.

El grupo toma su cuerpo en un espacio. El espacio en que se reúne es el lugar de despliegue de fantasías, límites, criterios, palabras e imaginarios. Los individuos que participan en un grupo comparten, según ibañez: el espacio vivido (cada uno con su correlato), el espacio real (el instituido para que funcione el grupo), el espacio imaginario (proyección de fantasías) y el espacio simbólico (construcción de un lugar que los articule a todos).

Con respecto al espacio simbólico, resulta muy interesante conocer lo que propone ibáñez en torno al carácter que un grupo asume cuando es ubicado en un espacio-tiempo que tiene sus límites y su duración. Los integrantes del grupo saben que no van a perdurar constituidos como grupo, son conscientes todos, de su transitoriedad y se asumen en estado de promesa, es decir, sienten que hacen grupo como posibilidad. Esta situación quizás incide en el discurso de cada participante pues las palabras van y vienen, de manera fluida, como si el espacio transitorio y la corta duración, determinarán el matiz de cada intervención.

A partir de la llegada de los participantes (actuantes) todo lo que ocurre es registrado como documento. El registro - señala ibáñez - es la huella del grupo, su escritura. Y es una escritura invisible que está hecha para el otro. Incluso puede suceder que los grupos de manera espontánea, decidan escribir sobre papel, tal vez dibujar o realizar intervenciones de carácter artístico. En general y más aún en el segundo grupo de discusión de este proyecto, los actuantes realizaron sus prácticas sobre papel y madera; entre tanto, en el primer grupo, los integrantes del Equipo Muitidisciplinario de Colombia organizaron la práctica artística titulada "Botánica de la comida", una cena con vegetales. En otras palabras, el grupo se reconoce como sujeto de un discurso y de una práctica. Generalmente, los grupos comienzan a hablar a partir de la intervención del preceptor, que es la figura que impulsa la discusión, provocando las Intervenciones y midiendo de manera continua el pulso y ritmo de la discusión. Después de la provocación inicial, vienen las preguntas que desencadenan la conversación durante el tiempo estimado para su duración. 
Frente a la situación observada en los tres grupos diseñados para la investigación, se puede afirmar que las discusiones ocurrieron en un tiempo límite estatuido por el preceptor. Cuatro horas de discusión en el primer grupo (una sola sesión); seis horas de discusión en el segundo grupo (dos sesiones durante dos días); y cuatro horas de discusión en el tercer grupo (una sola sesión). Afirma lbáñez que una vez terminada la discusión (y la reunión), el preceptor se encarga de reciclar mediante el análisis, los resultados de los encuentros.

Ciertamente, el preceptor se enfrenta al final con una gran cantidad de información: palabras, sonidos, gestualización, silencios, suspiros, pausas, momentos de tensión, rotación de la palabra y un sin fin de situaciones que de manera compleja dan consistencia a cada grupo y permiten reconocer que lo ocurrido, tiene una vitalidad que rompe cualquier predicción. Agrega Ibáñez:

"Para poner en orden la masa de datos (seleccionar los pertinentes e integrarlos en un espacio teórico unitario) el preceptor debe poner en marcha su intuición: La captación intuitiva, es decir, uno intuito, de la unidad inmediatamente perceptible de una situación, de un estilo de vida o de una manera de ser, conduce a indagar, en sus relaciones significantes, propiedades y relaciones que no se presentan sino sucesivamente en el trabajo analítico (...) esta capacidad de intuición es un precipitado de toda la experiencia y de todo el saber del investigador."

La metodología de grupos de discusión implementada ha tenido dos propósitos: El primero, recrear una manera distinta de investigación a partir de un escenario vivo que reúne distintos actores (actuantes) para conversar acerca de sus experiencias en una conversación fluida y directa; tal vez, la propuesta de diseñar estos grupos se refiera a la necesidad de buscar un lugar más flexible desde el cual pueda darse la interrelación entre la institución y los individuos. El segundo propósito ha sido producir un audiovisual y un texto escrito, que recoja la palabra viva y los elementos que configuran una situación discursiva; entre estos, la postura, los silencios, las pausas, las miradas y también, los objetos que rodean el discurso. Todos estos elementos y aspectos, recrean una especie de cinematografía que muestra cada vez en tiempo vivo, lo que acontece en cada grupo.

El siguiente párrafo de Ibáñez deja ver el espíritu de este experimento:

"Hay que saber perderse para trazar un mapa, salir de Ios caminos trillados, vagar: deambular por las encrucijadas, abrir senderos a través de las mieses y el desierto, penetrar en callejuelas sin salida; asumir que todo camino recorrido sin mapa es cático (luego será posible tender o recoger puentes, bordear pozos o simas, perforar agujeros o taparlos). $Y$ hay que saber subvertir la ley - y/0 acaso pervertirla - : apearse de todo lo dicho o lo sabido, quedar solo; hay que romper con todos los grupos, disentir de todos los consensos, hasta tocar la muerte 0 el silencio 8luego será otra vez posible confraternizar y conversar)"

El grupo es un espacio ficcional que produce otro tipo de discursos, menos rígidos y más blandos, singulares y al mismo tiempo colectivos pues interpelan todo el tiempo y son motores de su propio diálogo. Son discursos que no se inscriben en la lógica binaria y lineal sino que funcionan como sueño grupal, como mito, como símbolo de sociabilidad.

En todo caso, y para retomar el comienzo, tal vez convendría agregar la referencia de Hegel cuando dice que la filosofía llega siempre demasiado tarde (...) Cuando la filosofía pinta su grisalla en la grisalla -señala - una manifestación de la vida acaba de envejecer. No podemos rejuvenecerla poniendo gris sobre gris, sino sólo conocerla. La lechuza de Minerva solo emprende su vuelo al comienzo del crepúsculo" (Maffesoli, 1997, pág. 45). 


\section{PAIIEIA Suscolombiana 18}

\section{Bibliografía}

Agirre, Imanol. (2005). Teorías y prácticas en educación artística. Navarra: Octaedro.

Agudelo, César Ernesto. (2005). Subámonos al Colectivo. Colección de Ensayos sobre el Campo del Arte. Bogotá: Panamericana.

Arbeláez, María José. (2001). Educación, sociedad y creación. Pensamiento y creación artística. Bogotá: ASAB.

Bisquerra, A. R. (2009). Metodología de la Investigación Evaluativa. Madrid: La Muralla.

Brea, José Luis. (2005) Estudios Visuales. "La Epistemología de la Visualidad en la Era de la Globalización". Madrid: Akal.

Bourriaud, Nicolas. (2008). Estética Relacional. Buenos Aires: Adriana Hidalgo Editora.

Brea, José Luis. (2004). Estatuto de las prácticas artísticas en la era del capitalismo cultural. Murcia: CENDEAC.

Callejo, J. (2001). El grupo de discusión: introducción a una práctica de investigación. Barcelona: Ariel.

Danto, Arthur. (1997). Después del fin del Arte. Barcelona: Paidós.

Danto Arthur. (2005). Estéticas después del fin del arte. Madrid: La Balsa de la Medusa.

David. B. (20 de Diciembre de 2007). Teoría y Práxis Sociológica. Recuperado el Febrero de 2012, de http://teoriaypraxissociologica.wordpress.com/:

http://teoriaypraxissociologica.wordpress.com/2007/12/20/ibanez-y-el-grupo-de-discusion/

Ducrot, 0. y. (1972). Diccionario enciclopédico de las ciencias del lenguaje. Buenos Aires: Siglo XXI.

Eco, Humberto. (1972). La Definición del Arte. Barcelona: Martínez Roca.

Efland, Arthur C. Freedman, Kerry. Sthur, Patricia. (2003). La Educación en el Arte Postmoderno. Madrid: Paidós.

Graziano, Valeria. Intersecciones del arte, la cultura y el poder. Arte y teoría en el semiocapitalismo. (2005). En: Estudios Visuales. La Epistemología de la Visualidad en la Era de la Globalización. Madrid:Akal.

Fischer, Ernst. (1993). La necesidad del arte. Barcelona: Península-Nexos.

Gil, Javier. (2010). Pensamiento artístico y experiencia. Educación Artística y Cultural: Un propósito común. Cuadernos de Educación Artística. Bogotá: Ministerio de Cultura.

Hernández Sánchez, Domingo. (2002). Estéticas del Arte Contemporáneo. Madrid: Universidad Salamanca. Herrera Nelson. (2002). Coordenadas de arte contemporáneo. La Habana: Arte Cubano.

Ibañez, J. (1979). Más allá de la sociología. Madrid, España: Siglo XXI. 
Larrosa, J. (s.f.). http://es.scribd.com. Obtenido de Deseo de realidad:

http://es.scribd.com/doc/124686636/Deseo-de-Realidad-Jorge-Larrosa

Llopis G, R. (2004). El Grupo de Discusión, Manual de aplicación a la Investigación social, comercial y comunicativa. Madrid: ESIC.

Maffesoli, M. (1997). Elogio de la razón sensible. Barcelona: Paldós.

Maffesoli, Michel. (2007). En el Crisol de las Apariencias. Para una Ética de la Estética. Madrid: Siglo XXI.

Margott, Jean Paul. (1999). Modernidad, crisis de la modernidad y postmodernidad. Barranquilla: Uninorte.

Mandoky, Katia. (1994). Prosaica. Introducción a la Estéticas de lo Cotidiano. México: Grijalbo.

Martínez Miguelez, Miguel. (2007). Ciencia y Arte en la Metodología Cualitativa. México: Trillas.

Niño, Alejandra. (2009). Memoria e intersubjetividad en las prácticas artísticas contemporáneas. Universidad de Buenos Aires. En: AdVersuS. VI, 14,15, abril-agosto 2009.

Oliveras, Helena. (2005). Estética: La cuestión del arte. Buenos Aires: Ariel Filosofía.

Rancière, Jacques. (2010). El espectador emancipado. Buenos Aires: Bordes Manantial.

Sanabria, Carlos Eduardo. (2004) Estética. Miradas contemporáneas. Bogotá: Universidad de Jorge Tadeo Lozano.

Santos, Milton. (1993). Los espacios de la Globalización. En: Anales de Geografía de la Universidad Complutense No. 13,69-77 Madrid: Editorial Complutense.

UCM. (06 de 07 de 2010). ¿Qué son los grupos de discusión? Recuperado el febrero de 2012, de http://www.ucm.es/info/socivmyt/paginas/profesorado/falvira/Qu_es_un_grupo_de_discusi_n.doc., con acceso: 07-06-10:

http://www.google.com.co/url?sa=t\&rct=j\&q=\&esrc=s\&frm=1\&source=web\&cd=3\&sqi=2\&ved=0CDoQFjA C\&url=http\%3A\%2F\%2Fwww.ucm.es\%2Finfo\%2Fsocivmyt\%2Fpaginas\%2Fprofesorado\%2Ffalvira\%2FQu _es_un_grupo_de_discusi_n.doc\&ei=XRhoUeXzB4G08AT1ylGoDw\&usg=AFQjCNGic6TsJu39EL

Vanegas, Guillermo. (2005). Aprender a Discutir. Dinámicas de conversación en tres foros virtuales sobre arte contemporáneo en el campo artístico Colombiano 2000 - 2002. Colección de Ensayos sobre el Campo del Arte. Ensayos de Autor. Bogotá: Panamericana. 


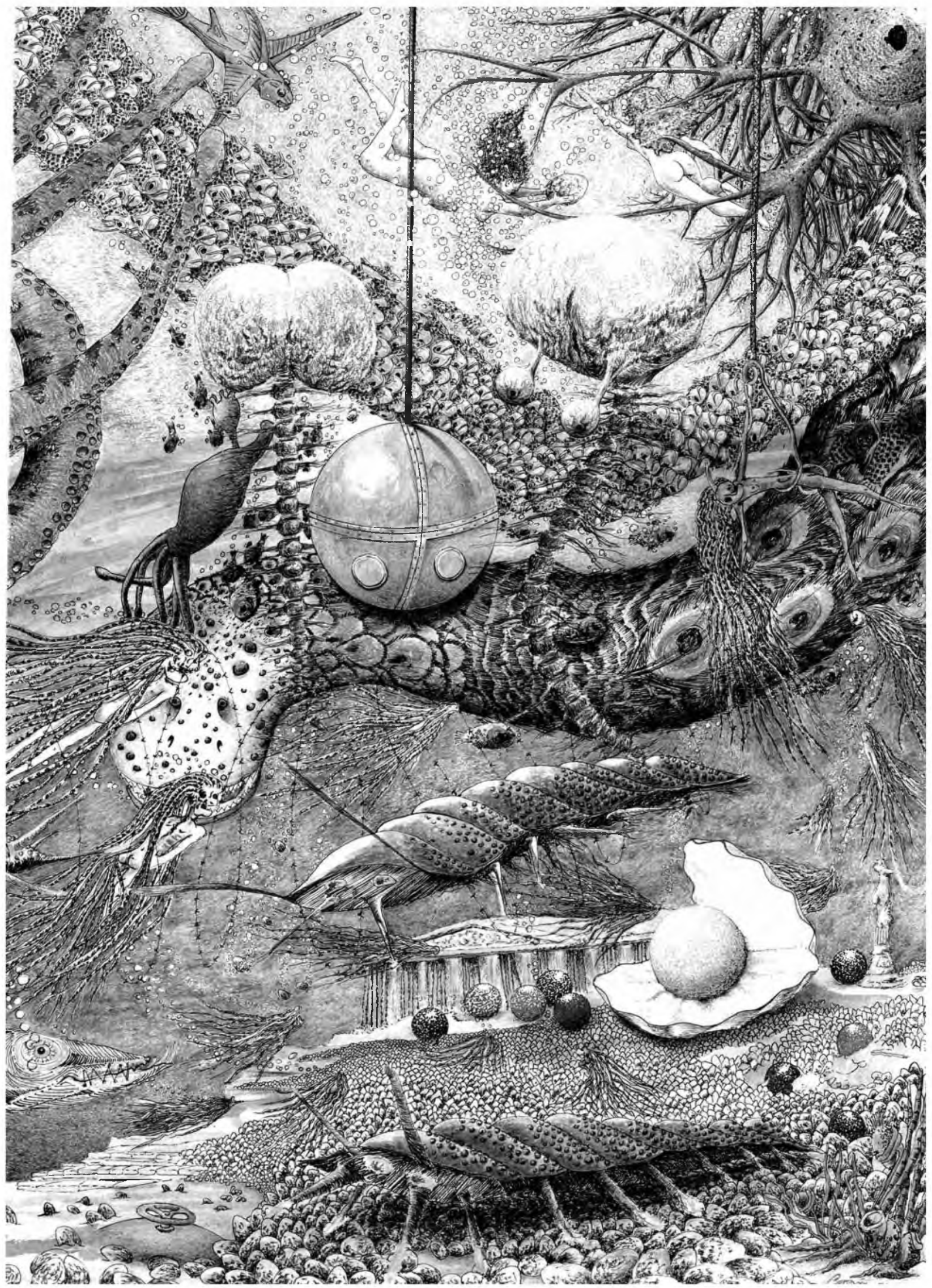

